\title{
Segmentation of Brain MR Images based on Finite Skew Gaussian Mixture Model with Fuzzy C-Means Clustering and EM Algorithm
}

\author{
Nagesh Vadaparthi \\ Department of I.T, \\ MVGR College of Engineering, \\ Vizianagaram, India
}

\author{
Suresh Varma Penumatsa \\ Dept. of Computer Science, \\ Adikavi Nannaya University, \\ Rajahmundry, India
}

\author{
Srinivas Yarramalle \\ Department of IT, \\ GITAM University, \\ Visakhapatnam, India
}

\author{
P.S.R.Murthy \\ Department of Mathematics, \\ GITAM University, \\ Visakhapatnam, India
}

\begin{abstract}
Segmentation is a process of converting inhomogeneous data into homogeneous data. There are many segmentation techniques available inthe literature. Among these techniques, finite Gaussian Mixture Model using EM algorithm is one mostly used. However, Gaussian Mixture Model is suited well when the image under consideration is symmetric. But in reality, medical images are asymmetric. Hence, it is needed to develop new algorithms for segmenting non - symmetric images. Therefore, skew symmetric mixture model is utilized for this purpose. The segmentation is carried out by using Fuzzy C-Means clustering technique and the updated parameters are obtained through EM algorithm. The model is tested with 8 images and the segmentation evaluation is carried out by using objective evaluation criteria namely Jaccard Coefficient (JC) and Volumetric Similarity (VS), Variation of Information (VOI), Global Consistency Error (GCE) and Probabilistic Rand Index (PRI). The performance evaluation of reconstructed images is carried out by using image quality metrics. The experimentation is carried out using $T_{1}$ weighted images and the results are compared with the existing models.
\end{abstract}

Keywords: Segmentation, Skew Gaussian Mixture Model, Objective Evaluation, Image Quality Metrics, EM algorithm

\section{INTRODUCTION}

The field of biomedical image analysis has emerged rapidly over the couple of decades. The wide spread availability of suitable detectors have helped for the latest developments in medical analysis with respect to monitoring, diagnosing and treatment of the patients. There are many methods available in the literature for the identification of the deformities with respect to the medical images pertaining to a patient's data. Among these methods, techniques based on segmentation and mixture models have gained popularity. Segmentation is mainly used by radiologists to segment the image into meaningful regions [1],[2],[3],[4].The specific application of these segmentation techniques is to detect the tumor regions by segmenting the MRI data [5]. The size of the tumor can be estimated by using the segmentation techniques thereby planning for the treatment. Many segmentation algorithms have been utilized for segmenting the brain images.Among these algorithms K-Means algorithm is mostly used. In KMeans clustering algorithm, the image is divided into number of homogeneous classes effectively and it works very efficiently if the image contains noise and the feature vectors of the dataset can be exactly a member of only one cluster. But, in medical images absolute classification of a pixel is not possible because of partial volume effects where multiple tissues contribute to a pixel or because a voxel causes intensity blurring across the boundaries and this type of clustering algorithms allows for the uncertainty in the location of the object boundaries. Moreover, the hierarchical clustering algorithm also shares similar arguments as the case of $\mathrm{K}$ Means algorithm. In order to segment the medical image more approximately, Fuzzy C-Means algorithm is widely preferred because of the additional flexibility which allows the pixel to belong to multiple classes with varying degree of membership [6]. Hence, in this paper, Finite Skew Gaussian distribution mixture model with Fuzzy C-Means clustering algorithm is proposed to segment the image into number of image regions. The performance of the segmentation algorithm is carried out by using objective evaluation metrics such as Jaccard Coefficient (JC), Volumetric Similarity (VS), Variation of Information (VOI), Global Consistency Error (GCE) and Probabilistic Rand Index (PRI). The performance of developed medical image segmentation algorithm is compared with Finite Skew Gaussian Mixture Model with KMeans algorithm and with Finite Skew Gaussian Mixture Model with Hierarchical Clustering algorithm using quality metrics such as Average Difference, Maximum Distance, Image Fidelity, Mean squared error and Signal to noise ratio. The accuracy of the developed algorithm is tested with brain medical images.

The initial estimates obtained by Fuzzy C-Means clustering are refined by using EM algorithm presented in section -5 of the paper. The paper is organized as follows: Section -2 deals with introduction to Fuzzy C-Means clustering. Section -3 deals with Skew Gaussian Distribution, initialization of parameters is discussed in section -4 and the updation of initial estimates is presented in section -5 . Section- 6 explains about the segmentation algorithm and in section-7, experimental results and performance of the algorithm is discussed. Finally, section- 8 concludes the paper. The 
experimentation is carried out with 8 different medical images and the results are tabulated.

\section{FUZZY C-MEANS CLUSTERING ALGORITHM}

The first step in any segmentation algorithm is to divide image into different image regions. Many segmentation algorithms are presented in literature [6],[7],[8],[9],[10]. Among these techniques, medical image segmentation based on K-Means is mostly utilized [5]. But, the main disadvantage with K-Means is that, K-Means are slow in convergence and pseudo unsupervised learning that requiresthe initial value of K. Apart from K-Means, hierarchical clustering algorithm is also used but even this algorithm shares similar arguments as the case of K-Means algorithm. Hence, in this paper we have used Fuzzy C-Means clustering algorithm inorder to identify the initial clusters. The algorithm for Fuzzy C-means clustering is presented below.

The FCM employs fuzzy partitioning such that a data point can belong to all groups with different membership grades between 0 and 1 and it is an iterative algorithm. The aim of FCM is to find cluster centers (centroids) that minimize a dissimilarity function.To accommodate the introduction of fuzzy partitioning, the membership matrix (U) is randomly initialized according to Equation (1).

$\sum_{i=1}^{c} u_{i j}=1, \forall j=1, \ldots, n$

The dissimilarity function which is used in FCM is given Equation (2)

$J\left(U, c_{1}, c_{2}, \ldots, c_{c}\right)=\sum_{i=1}^{c} J_{i}=\sum_{i=1}^{c} \sum_{j=1}^{n} u_{i j}{ }^{m} d_{i j}{ }^{2}$

Where, $\mathrm{u}_{\mathrm{ij}}$ is between 0 and 1 ;

$c_{i}$ is the centroid of cluster $i$

and $\mathrm{j}_{\text {th }}$ data point;

$\mathrm{d}_{\mathrm{ij}}$ is the Euclidian distance between $\mathrm{i}_{\text {th }}$ centroid $\left(\mathrm{c}_{\mathrm{i}}\right)$

$\mathrm{m} \epsilon[1, \infty]$ is a weighting exponent.

To reach a minimum of dissimilarity function there are two conditions. These are given in Equation (3) and Equation (4).

$c_{i}=\frac{\sum_{j=1}^{n} u_{i j}{ }^{m} x_{j}}{\sum_{j=1}^{n} u_{i j}{ }^{m}}$

$u_{i j}=\frac{1}{\sum_{k=1}^{c}\left(\frac{d_{i j}}{d_{k j}}\right)^{2 /(m-1)}}$

This algorithm determines the following steps.

Step-1:Randomly initialize the membership matrix (U) that has constraints in Equation (1).

Step-2:Calculate centroids $\left(\mathrm{c}_{\mathrm{i}}\right)$ by using Equation (3).

Step-3:Compute dissimilarity between centroids and data points using equation (2). Stop if its improvement over previous iteration is below a threshold.
Step-4:Compute a new U using Equation (4). Go to Step 2.

By iteratively updating the cluster centers and the membership grades for each data point, FCM iteratively moves the cluster centers to the "right" location within a data set.

FCM does not ensure that it converges to an optimal solution. Because of cluster centers (centroids) are initialize using U that randomly initialized.(Equation (3)).

Performance depends on initial centroids. For a robust approach there are two ways which is described below.

1) Using an algorithm to determine all of the centroids. (for example: arithmetic means of all data points)

2) Run FCM several times each starting with different initial centroids.

\section{SKEW GAUSSIAN DISTRIBUTION}

The pixels intensities inside the medical images may not be symmetric or bell shaped due to several factors associated like part of the body, bone structure etc. In these cases, the pixels are distributed asymmetrically and follow a skew distribution. Hence, to categorize these sorts of medical images, Skew Gaussian distribution is well suited. Every image is a collection of several regions. To model the pixel intensities inside these image regions, we assume that the pixels in each region follow a Skew normal distribution, where the probability density function is given by

$f(z)=2 . \emptyset(z) . \Phi(\propto z) ; \quad-\infty<z<\infty$.

where, $\Phi(\propto \mathrm{z})=\int_{-\infty}^{\alpha z} \emptyset(t) d t$.

and, $\quad \varnothing(z)=\frac{e^{-\frac{1}{2} z^{2}}}{\sqrt{2 \pi}}$.

Let, $\quad y=\mu+\sigma z$

$$
z=\frac{y-\mu}{\sigma} .
$$

Substituting equations (6), (7), and (8) in equation (5),

$f(z)=\sqrt{\frac{2}{\pi}} . \quad e^{-\frac{1}{2}\left(\frac{y-\mu}{\sigma}\right)^{2}}\left[\int_{-\infty}^{\alpha\left(\frac{y-\mu}{\sigma}\right)} \frac{e^{-\frac{1}{2}\left(\frac{t-\mu}{\sigma}\right)^{2}}}{\sqrt{2 \pi}} d t\right]$.

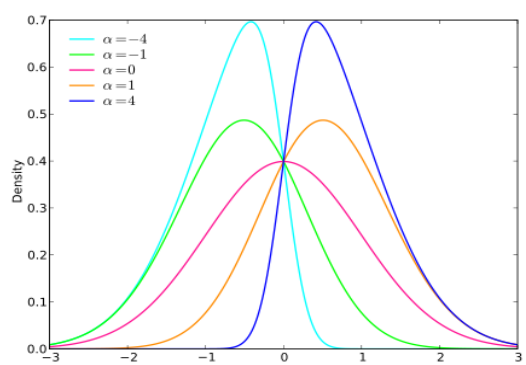

Figure-1:Skew Normal Distributions

\section{INITIALIZATION OF PARAMETERS}

In order to initialize the parameters, it is needed to obtain the initial values of the model distribution. The initial estimates of the Mixture model $\mu_{\mathrm{i}}, \sigma_{\mathrm{i}}$ and $\alpha_{\mathrm{i}}$ where $\mathrm{i}=1,2, \mathrm{k}$ are estimated using Hierarchical Clustering algorithm as proposed in section-II. It is assumed that the pixel intensities of the entire 
image is segmented into a $\mathrm{K}$ component model $\pi_{\mathrm{i}}, \mathrm{i}=1,2 \ldots \mathrm{K}$ with the assumption that $\pi_{\mathrm{i}}=1 / \mathrm{K}$ where $\mathrm{K}$ is the value obtained from Hierarchical Clustering algorithm discussed in section-II

\section{UPDATION OF INITIAL ESTIMATES THROUGH EM ALGORITHM}

The initial estimates of $\mu_{\mathrm{i}}{ }^{1+1}, \sigma_{\mathrm{i}}{ }^{1+1}, \alpha_{\mathrm{i}}{ }^{1+1}$ that are obtained from section -4 are to be refined to obtain the final estimates. For this purpose EM algorithm is utilized. The EM algorithm consists of 2 steps E-step and M-Step. In the E-Step, the initial estimates obtained in section -4 are taken as input and the final updated equations are obtained in the M-Step. The updated equations for the model parameters $\mu, \sigma$ and $\alpha$ are given below.

$$
\begin{aligned}
& \mu^{(1+1)}=
\end{aligned}
$$

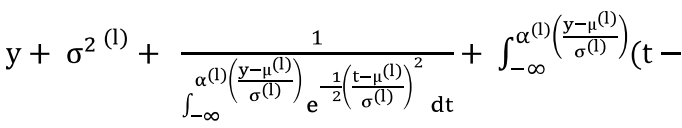

$$
\begin{aligned}
& \left.\mu^{(1)}\right) \mathrm{e}^{-\frac{1}{2}\left(\frac{\mathrm{t}-\mu^{(1)}}{\sigma^{(1)}}\right)^{2}} \mathrm{dt}-\sigma^{(1)} \alpha^{(1)} \mathrm{e}^{\frac{\left[\left(\alpha^{(1)}+\sigma^{(1)}\right) \mu^{(1)}-\alpha^{(1)} \mathrm{y}\right]^{2}}{2 \sigma^{4^{(l)}}}} \\
& \sigma^{(1+1)}=1
\end{aligned}
$$

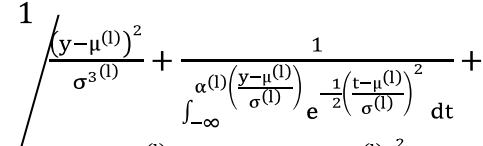

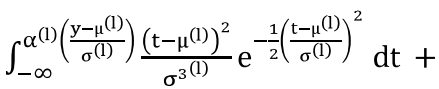

$$
\begin{aligned}
& \alpha^{(\mathrm{l})}\left(\frac{\mu^{(1)}-\mathrm{y}}{\left.\sigma^{2^{(1)}}\right)} \mathrm{e}^{\frac{\left[\left(\alpha^{(1)}+\sigma^{(1)}\right) \mu^{(1)}-\alpha^{(1)} y\right]^{2}}{2 \sigma^{4^{(1)}}}}\right. \\
& \alpha^{(l+1)}=\frac{\sqrt{2} \sigma^{2}(l)}{\mu^{(l)}-y}\left[\log \left(\int_{-\infty}^{\alpha^{(l)}\left(\frac{y-\mu(l)}{\sigma^{(l)}}\right)} e^{-\frac{1}{2}\left(\frac{t-\mu^{(l)}}{\sigma^{(l)}}\right)^{2}} d t\right)-\right. \\
& \left.\log \left(\frac{y-\mu^{(l)}}{\sigma^{(l)}}\right)\right]^{\frac{1}{2}}-\frac{\sigma^{(l)} \mu^{(l)}}{\mu^{(l)}-y}
\end{aligned}
$$

\section{SEGMENTATION ALGORITHM}

After refining the parameters, the first step in image reconstruction by allocating pixels to the segments. This operation is done by the segmentation algorithm. The segmentation algorithm consists of 7 steps.

Step 1: Obtain the pixel intensities of the gray image. Let they be represented by $\mathrm{x}_{\mathrm{ij}}$.

Step 2: Obtain the number of regions by k-means algorithm and divide the (image) pixelinto regions.

Step 3: For each region obtain the initial estimates using moment methods of estimationfor $\mu_{\mathrm{i}}, \sigma_{\mathrm{i}}$. Let $\alpha_{\mathrm{i}}=1 / \mathrm{k}$ be the initial estimate for $\alpha_{\mathrm{i}}$

Step 4: Obtain the refined estimates of $\mu_{i}, \sigma_{i}, \alpha_{i}$ for $i=1 \ldots k$ using updated equations forthe parameters derived by EM algorithm with step 3 estimates as initial estimates.
Step 5: Implement the segmentation and retrieval algorithm by considering maximum likelihood estimate.

Step 6: With the step 5 obtain the image quality metric.

Step 7:The image segmentation is carried out by assigning each pixel into a properregion (Segment) according to maximum likelihood estimates of the $j^{\text {th }}$ element $L_{j}$ according to the following equation

$$
\mathrm{L}_{\mathrm{j}}=\operatorname{Max}_{\mathrm{j}}\left\{\sqrt{\frac{2}{\pi}} . \quad e^{-\frac{1}{2}\left(\frac{y-\mu}{\sigma}\right)^{2}}\left[\int_{-\infty}^{\alpha\left(\frac{y-\mu}{\sigma}\right)} \frac{e^{-\frac{1}{2}\left(\frac{t-\mu}{\sigma}\right)^{2}}}{\sqrt{2 \pi}} d t\right]\right\}
$$

\section{EXPERIMENTAL RESULTS AND PERFORMANCE EVALUATION}

After developing the segmentation algorithm, the algorithm is applied to8 different medical images obtained from the web database. The segmentation performance is evaluated by using objective segmentation evaluation criteria based on Jaccard Index and Volumetric similarity using formulas

$$
\begin{aligned}
& \mathrm{JC}=\frac{|X \cap Y|}{|X \cup Y|}=\frac{\mathrm{a}}{\mathrm{a}+\mathrm{b}+\mathrm{c}} \\
& \mathrm{VC}=1-\frac{|\mathrm{X}|-|\mathrm{Y}| \mid}{|\mathrm{X}|+|\mathrm{Y}|}=1-\frac{|\mathrm{b}-\mathrm{c}|}{2 \mathrm{a}+\mathrm{b}+\mathrm{c}}
\end{aligned}
$$

Where, $a=|X \cap Y|, b=\left|\frac{X}{Y}\right|, \mathrm{c}=\left|\frac{Y}{X}\right|, \mathrm{d}=|\overline{X \cup Y}|$

$\operatorname{GCE}\left(\mathrm{S}, \mathrm{S}^{\prime}\right)=\frac{1}{\mathrm{~N}} \min \left\{\sum \operatorname{LRE}\left(\mathrm{S}, \mathrm{S}^{\prime}, \mathrm{x}_{\mathrm{i}}\right), \sum \operatorname{LRE}\left(\mathrm{S}^{\prime}, \mathrm{S}, \mathrm{x}_{\mathrm{i}}\right)\right\}(15)$

Where, $\mathrm{LRE}=\frac{\left|C\left(S, \mathrm{x}_{\mathrm{i}}\right) \backslash C\left(S^{\prime}, \mathrm{x}_{\mathrm{i}}\right)\right|}{\left|C\left(S, \mathrm{x}_{\mathrm{i}}\right)\right|}$

$\mathrm{S}$ and $\mathrm{S}^{\prime}$ are segment classes and $\mathrm{x}_{\mathrm{i}}$ is the pixel.

$$
\operatorname{VOI}(X, Y)=H(X)=H(Y)-2 I(X ; Y)
$$

Where, $\mathrm{X}$ and $\mathrm{Y}$ are two clusters

$\operatorname{PRI}\left(\mathrm{S}_{t},\{\mathrm{~S}\}\right)=\frac{1}{\left(\begin{array}{c}N \\ 2\end{array}\right)} \sum_{i, j, i<j}\left[I\left(l_{i}^{S_{t}}=l_{j}^{S_{t}}\right) p_{j}+I\left(l_{i}^{S_{t}} \neq l_{j}^{S_{t}}\right)(1-\right.$ $\left.\left.p_{j}\right)\right]$

Where, $p_{j}=P\left(l_{i}=l_{j}\right)=\frac{1}{K} \sum_{k=1}^{K} I\left(l_{i}^{k}=l_{j}^{k}\right)$ and the values range from 0 to 1.1 denotes the segments are identical.

And the results obtained are tabulated in Table - I \& Figure I and the same is depicted using the bar graphs - I 
Table - 1: Segmentation Metrics

\begin{tabular}{|c|c|c|c|c|c|c|c|}
\hline Image & $\begin{array}{l}\text { Quality } \\
\text { Metric }\end{array}$ & GMM & $\begin{array}{c}\text { Skew } \\
\text { GMM } \\
\text { with k- } \\
\text { Means- } \\
\text { EM }\end{array}$ & $\begin{array}{c}\text { Skew GMM } \\
\text { with Hierar. } \\
\text { Clustering- } \\
\text { EM }\end{array}$ & $\begin{array}{c}\text { Skew } \\
\text { GMM } \\
\text { with FCM } \\
\text {-EM }\end{array}$ & $\begin{array}{c}\text { Standard } \\
\text { Limits }\end{array}$ & Standard Critrial \\
\hline \multirow{5}{*}{ B0S1 } & $\mathrm{JC}$ & 0.089 & 0.689 & 0.703 & 0.795 & 0 to 1 & Close to 1 \\
\hline & VS & 0.432 & 0.733 & 0.8799 & 0.891 & 0 to 1 & Close to 1 \\
\hline & VOI & 2.3665 & 5.3173 & 5.142 & 5.232 & $-\infty$ to $\infty$ & As big as Possible \\
\hline & GCE & 0.2802 & 0.5964 & 0.561 & 0.4223 & 0 to 1 & Close to 1 \\
\hline & PRI & 0.504 & 0.6396 & 0.619 & 0.7958 & 0 to 1 & Close to 1 \\
\hline \multirow{5}{*}{ B0S2 } & $\mathrm{JC}$ & 0.0677 & 0.7656 & 0.7921 & 0.819 & 0 to 1 & Close to 1 \\
\hline & VS & 0.3212 & 0.8767 & 0.8814 & 0.8914 & 0 to 1 & Close to 1 \\
\hline & VOI & 1.9724 & 3.924 & 4.35 & 6.2894 & $-\infty$ to $\infty$ & As big as Possible \\
\hline & GCE & 0.2443 & 0.4741 & 0.419 & 0.4664 & 0 to 1 & Close to 1 \\
\hline & PRI & 0.416 & 0.5016 & 0.514 & 0.6847 & 0 to 1 & Close to 1 \\
\hline \multirow{5}{*}{ B0S3 } & $\mathrm{JC}$ & 0.0434 & 0.6567 & 0.7143 & 0.784 & 0 to 1 & Close to 1 \\
\hline & VS & 0.123 & 0.812 & 0.916 & 0.926 & 0 to 1 & Close to 1 \\
\hline & VOI & 0.7684 & 0.2916 & 1.659 & 5.5318 & $-\infty$ to $\infty$ & As big as Possible \\
\hline & GCE & 0.089 & 0.031 & 0.107 & 0.4001 & 0 to 1 & Close to 1 \\
\hline & PRI & 0.576 & 0.5853 & 0.632 & 0.706 & 0 to 1 & Close to 1 \\
\hline \multirow{5}{*}{ B0S4 } & $\mathrm{JC}$ & 0.0456 & 0.7878 & 0.874 & 0.911 & 0 to 1 & Close to 1 \\
\hline & VS & 0.2233 & 0.3232 & 0.54 & 0.643 & 0 to 1 & Close to 1 \\
\hline & VOI & 1.268 & 1.569 & 3.354 & 4.1619 & $-\infty$ to $\infty$ & As big as Possible \\
\hline & GCE & 0.056 & 0.091 & 0.157 & 0.2949 & 0 to 1 & Close to 1 \\
\hline & PRI & 0.189 & 0.191 & 0.496 & 0.5628 & 0 to 1 & Close to 1 \\
\hline \multirow{5}{*}{ B1S1 } & $\mathrm{JC}$ & 0.141 & 0.776 & 0.791 & 0.826 & 0 to 1 & Close to 1 \\
\hline & VS & 0.313 & 0.397 & 0.784 & 0.7910 & 0 to 1 & Close to 1 \\
\hline & VOI & 1.6499 & 4.0874 & 3.951 & 4.4115 & $-\infty$ to $\infty$ & As big as Possible \\
\hline & GCE & 0.1874 & 0.4487 & 0.418 & 0.2752 & 0 to 1 & Close to 1 \\
\hline & PRI & 0.9256 & 0.6678 & 0.6258 & 0.686 & 0 to 1 & Close to 1 \\
\hline \multirow{5}{*}{ B1S2 } & $\mathrm{JC}$ & 0.098 & 0.7892 & 0.877 & 0.896 & 0 to 1 & Close to 1 \\
\hline & VS & 0.04334 & 0.878 & 0.881 & 0.918 & 0 to 1 & Close to 1 \\
\hline & VOI & 2.3215 & 2.8047 & 3.91 & 6.6411 & $-\infty$ to $\infty$ & As big as Possible \\
\hline & GCE & 0.2838 & 0.3407 & 0.339 & 0.4661 & 0 to 1 & Close to 1 \\
\hline & PRI & 0.3807 & 0.369 & 0.485 & 0.6322 & 0 to 1 & Close to 1 \\
\hline \multirow{5}{*}{ B1S3 } & $\mathrm{JC}$ & 0.0222 & 0.8926 & 0.9124 & 0.946 & 0 to 1 & Close to 1 \\
\hline & VS & 0.3223 & 0.3429 & 0.3543 & 0.3869 & 0 to 1 & Close to 1 \\
\hline & VOI & 1.2411 & 0.9988 & 2.665 & 6.7129 & $-\infty$ to $\infty$ & As big as Possible \\
\hline & GCE & 0.1466 & 0.1157 & 0.398 & 0.4559 & 0 to 1 & Close to 1 \\
\hline & PRI & 0.9576 & 0.9662 & 0.652 & 0.7202 & 0 to 1 & Close to 1 \\
\hline \multirow{5}{*}{ B1S4 } & $\mathrm{JC}$ & 0.455 & 0.762 & 0.815 & 0.854 & 0 to 1 & Close to 1 \\
\hline & VS & 0.329 & 0.7001 & 0.7158 & 0.786 & 0 to 1 & Close to 1 \\
\hline & VOI & $-8.8 \mathrm{e}-16$ & 0 & 0.19 & 5.0898 & $-\infty$ to $\infty$ & As big as Possible \\
\hline & GCE & 0 & 0 & 0.212 & 0.3062 & 0 to 1 & Close to 1 \\
\hline & PRI & 1 & 1 & 0.27 & 0.5573 & 0 to 1 & Close to 1 \\
\hline
\end{tabular}

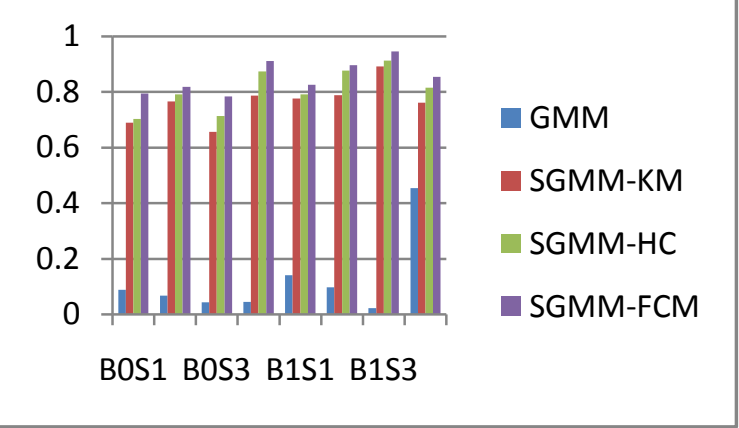

(A) Jaccard Coefficient

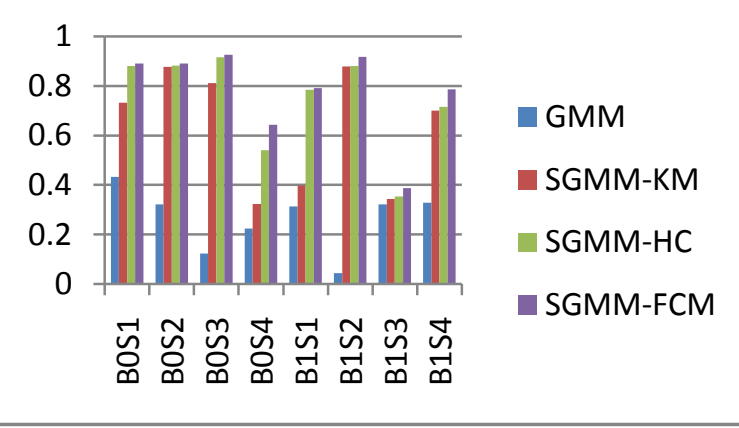

(B) Volume Similarity 


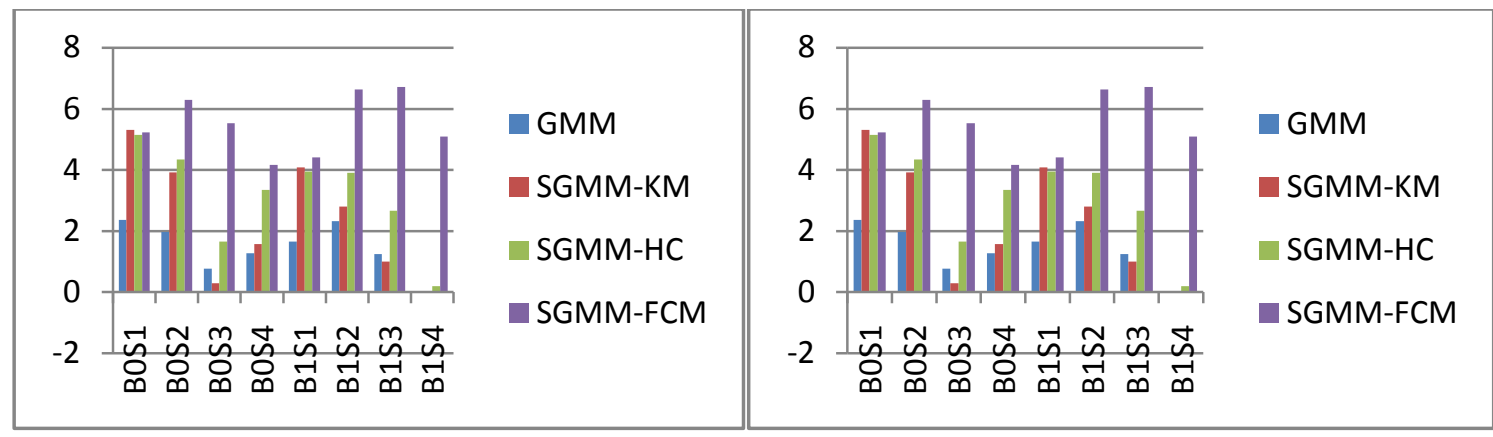

(C) Variation of Information

(D) Global Consistency Error

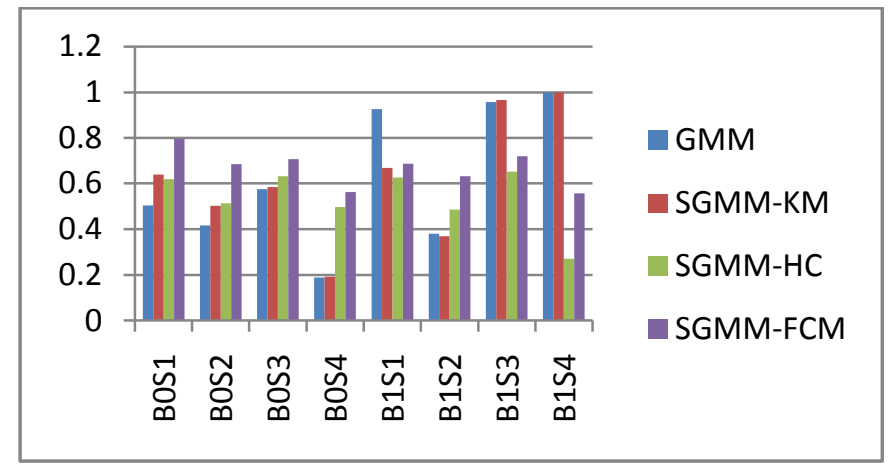

(E) Probabilistic Rand Index

Graph - 1: Comparison of Image Segmentation Techniques

In order to demonstrate the algorithm, the initial number of segments of the medical images under consideration is obtained from the histograms of the respective image and is presented in Table -2 .

TABLE - 2: Initial Estimates of K(By Histogram)

\begin{tabular}{|c|c|c|}
\hline Image & B2 & B3 \\
\hline Estimation for K & 4 & 3 \\
\hline
\end{tabular}

After obtaining the initial estimates, hierarchical clustering is applied for obtaining initial estimates of model parameters and initial estimates of number of segments for each of medical image and is presented in Table -3 .
TABLE - 3: Estimates of Hierarchical Clustering

\begin{tabular}{|l|c|c|}
\hline \multicolumn{1}{|c|}{ Image } & B2 & B3 \\
\hline $\begin{array}{l}\text { Estimateof Hierarchical } \\
\text { Clustering }\end{array}$ & 4 & 4 \\
\hline
\end{tabular}

After obtaining the initial estimates, the equations for EM algorithm are derived and the final parameters are estimated and are presented in Table -4 .

Table -4 : Estimation of initial and final parameters

\begin{tabular}{|c|c|c|c|c|c|c|c|}
\hline \multirow{3}{*}{ Image } & \multirow{3}{*}{$\begin{array}{l}\text { Regions } \\
\text { (i) }\end{array}$} & \multicolumn{3}{|c|}{ Estimation of initial parameters } & \multicolumn{3}{|c|}{$\begin{array}{l}\text { Estimation of final parameters using } \\
\text { EM algorithm }\end{array}$} \\
\hline & & \multicolumn{3}{|c|}{ Number of image regions, $k=4$} & \multicolumn{3}{|c|}{ Number of image regions, $k=4$} \\
\hline & & $\mu_{i}$ & $\sigma_{\mathrm{i}}$ & $\boldsymbol{\alpha}_{\mathrm{i}}$ & $\mu_{i}{ }^{\prime}$ & $\sigma_{i}{ }^{\prime}$ & $\boldsymbol{\alpha}_{i}{ }^{\prime}$ \\
\hline \multirow{4}{*}{ B1 } & S1 & 6.7126 & 10.247 & 0.3 & 0.0865 & 0.821 & 0.3 \\
\hline & S2 & 61.73 & 16.89 & 0.3 & 0.0002 & 0.004 & 0.3 \\
\hline & S3 & 123.55 & 22.37 & 0.2 & $6.41 \mathrm{e}-05$ & 0.0024 & 0.2 \\
\hline & S4 & 214.59 & 24.97 & 0.2 & $4.421 \mathrm{e}-05$ & 0.0013 & 0.2 \\
\hline
\end{tabular}

\begin{tabular}{|c|c|c|c|c|c|c|c|}
\hline \multirow{3}{*}{ Image } & \multirow{3}{*}{$\begin{array}{l}\text { Regions } \\
\text { (i) }\end{array}$} & \multicolumn{3}{|c|}{ Estimation of initial parameters } & \multicolumn{3}{|c|}{$\begin{array}{l}\text { Estimation of final parameters using } \\
\text { EM algorithm }\end{array}$} \\
\hline & & \multicolumn{3}{|c|}{ Number of image regions, $k=4$} & \multicolumn{3}{|c|}{ Number of image regions, $k=4$} \\
\hline & & $\mu_{\mathrm{i}}$ & $\sigma_{\mathbf{i}}$ & $\alpha_{i}$ & $\mu_{\mathrm{i}}^{\prime}$ & $\sigma_{\mathrm{i}}^{\prime}$ & $\alpha_{i}^{\prime}$ \\
\hline \multirow{4}{*}{ B2 } & S1 & 3.64 & 8.23 & 0.3 & -0.4891 & 0.949 & 0.3 \\
\hline & S2 & 51.08 & 16.31 & 0.3 & $8.203 \mathrm{e}-11$ & $1.16 \mathrm{e}-09$ & 0.3 \\
\hline & S3 & 115.46 & 18.62 & 0.2 & $6.512 \mathrm{e}-11$ & $9.05 \mathrm{e}-10$ & 0.2 \\
\hline & $\mathrm{S} 4$ & 179.8 & 24.86 & 0.2 & 3.3022 & 13.198 & 0.2 \\
\hline
\end{tabular}


After obtaining the updated estimates, using these estimates the image reconstruction is carried out byassigning each pixel in the PDF of the image and the outputs obtained are presented below in figure-2.

The image reconstruction is carried out by assigning each pixel to the segments using the segmentation algorithm and the probability density function and is given as follows. $f(z)=\sqrt{\frac{2}{\pi}} \cdot \quad e^{-\frac{1}{2}\left(\frac{y-\mu}{\sigma}\right)^{2}}\left[\int_{-\infty}^{\alpha\left(\frac{y-\mu}{\sigma}\right)} \frac{e^{-\frac{1}{2}\left(\frac{t-\mu}{\sigma}\right)^{2}}}{\sqrt{2 \pi}} d t\right]$

After reconstructing the image, the reconstructed images are shown below.

Figure - 2: Input and Reconstructed images using SGMM - HC

\begin{tabular}{|c|c|c|}
\hline Image & Original Image & Reconstructed Image \\
\hline B0S1 & & \\
\hline B0S2 & & \\
\hline B0S3 & & \\
\hline B0S4 & & \\
\hline B1S1 & & \\
\hline $\mathrm{B} 1 \mathrm{~S} 2$ & & \\
\hline B1S3 & & \\
\hline B1S4 & & \\
\hline
\end{tabular}

In order to evaluate the performance of the reconstructed image, image quality metrics are used and the metrics utilized for this purpose are presented in below table- 5 . 
Table- 5: Formulae for Evaluating Quality Metrics Used

\begin{tabular}{|l|c|}
\hline Quality metric & Formula to Evaluate \\
\hline Average Difference & $\sum_{j=1}^{M} \sum_{k=1}^{N}[F(j, k)-\hat{F}(j, k)] / M N$ \\
& Where M,N are image matrix rows and columns \\
\hline Maximum Distance & $\operatorname{Max}\{|F(j, k)-\hat{F}(j, k)|\}$ \\
\hline Image Fidelity & $\left.1-\left[\sum_{j=1}^{M} \sum_{k=1}^{N}[F(j, k)-\hat{F}(j, k)]^{2} / \sum_{j=1}^{M} \sum_{k=1}^{N}[F(j, k))\right]^{2}\right]$ \\
\hline & $\frac{1}{M N} \sum_{j=1}^{M} \sum_{k=1}^{N}[O\{F(j, k)\}-O\{\hat{F}(j, k)\}]^{2} / \sum_{j=1}^{M} \sum_{k=1}^{N}\left[O\left\{F(j, k)^{2}\right.\right.$ \\
Mean Squared error & Where M,N are image matrix rows and columns \\
\hline $\begin{array}{c}\text { Peak Signal to noise } \\
\text { ratio }\end{array}$ & \begin{tabular}{c} 
Whe log ${ }_{10}\left(\frac{M A X_{I}}{\sqrt{M S E}}\right)$ \\
\hline
\end{tabular} \\
\hline
\end{tabular}

Using above metrics, the performance evaluation is carried out and the comparison is done with respect to the model proposed using skew symmetric distribution [5] and the results are presented below in Table -5 and bar graphs -2 .

TABLE - 6: QUALITY MEASURES

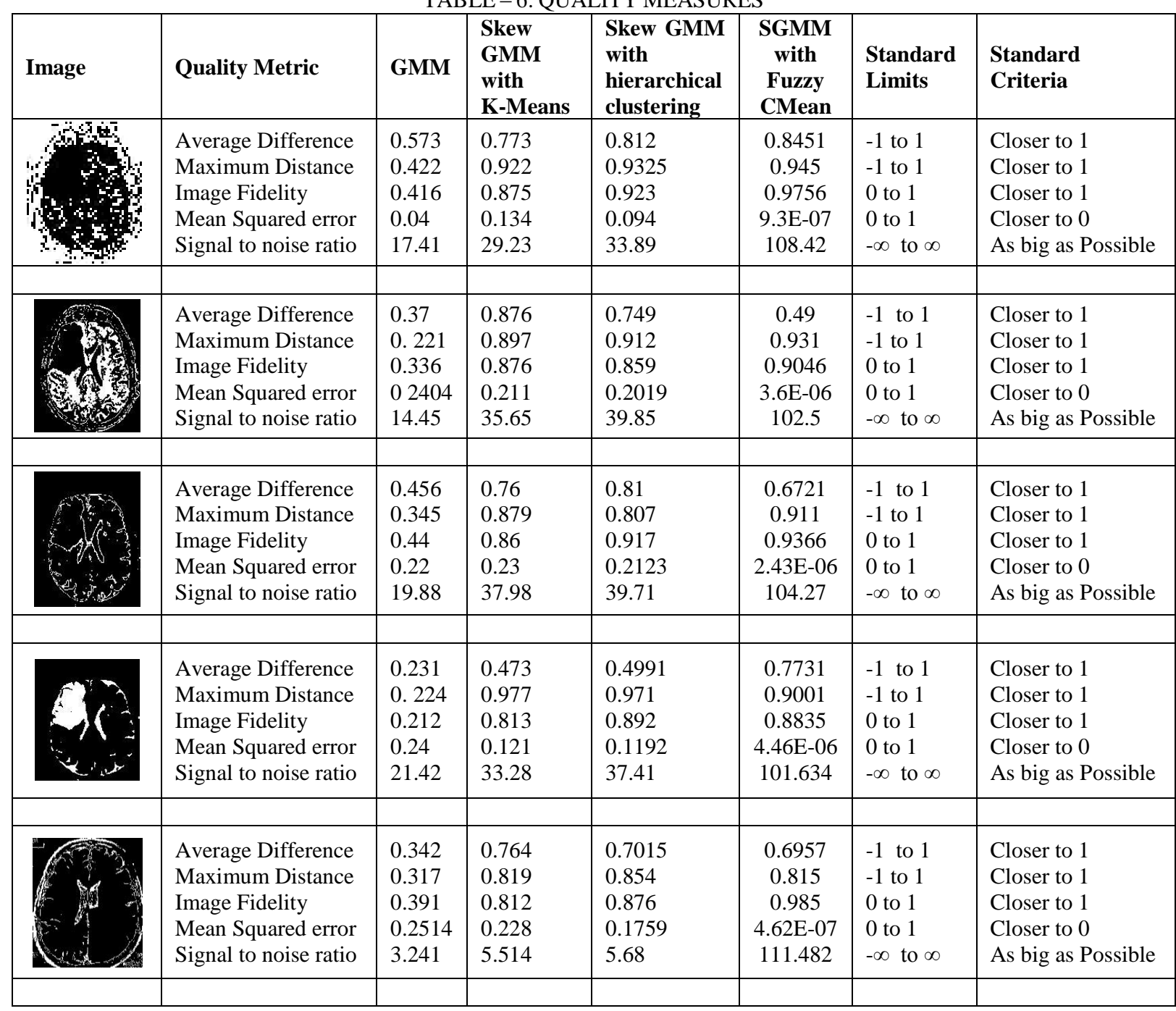




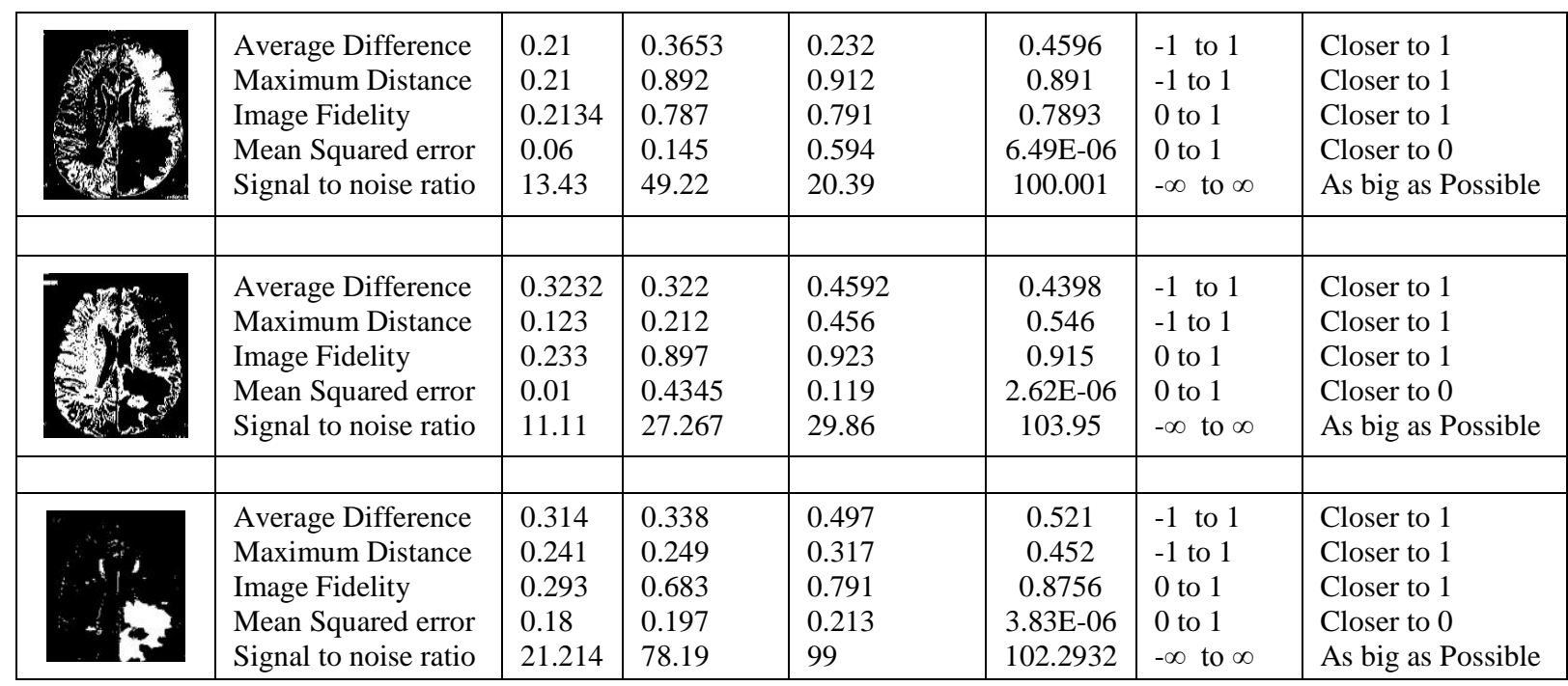

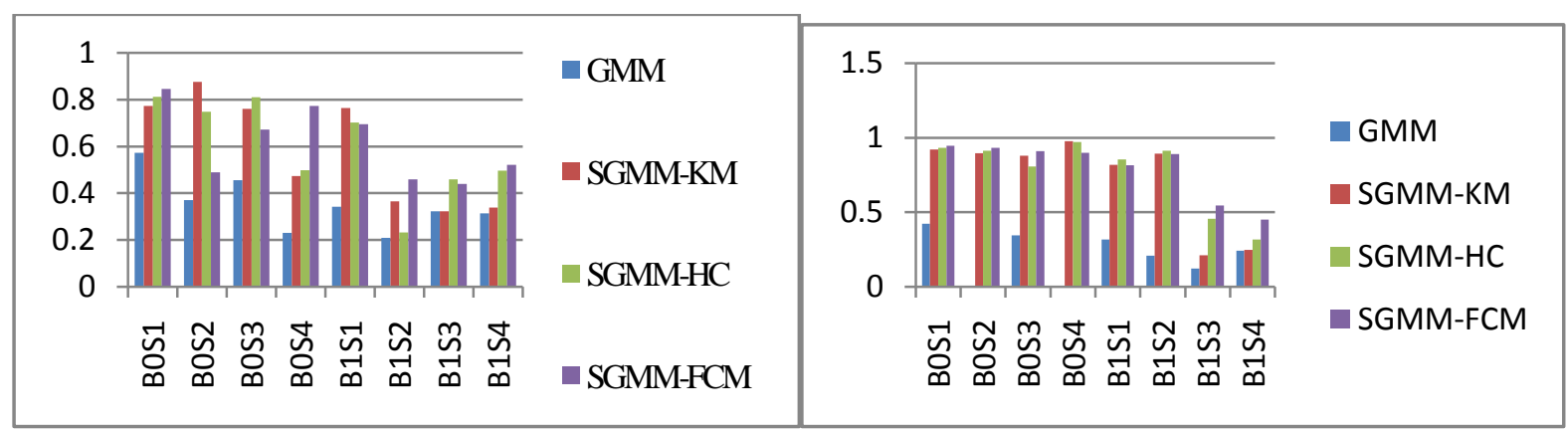

Figure A : Average Difference

Figure B: Maximum Distance

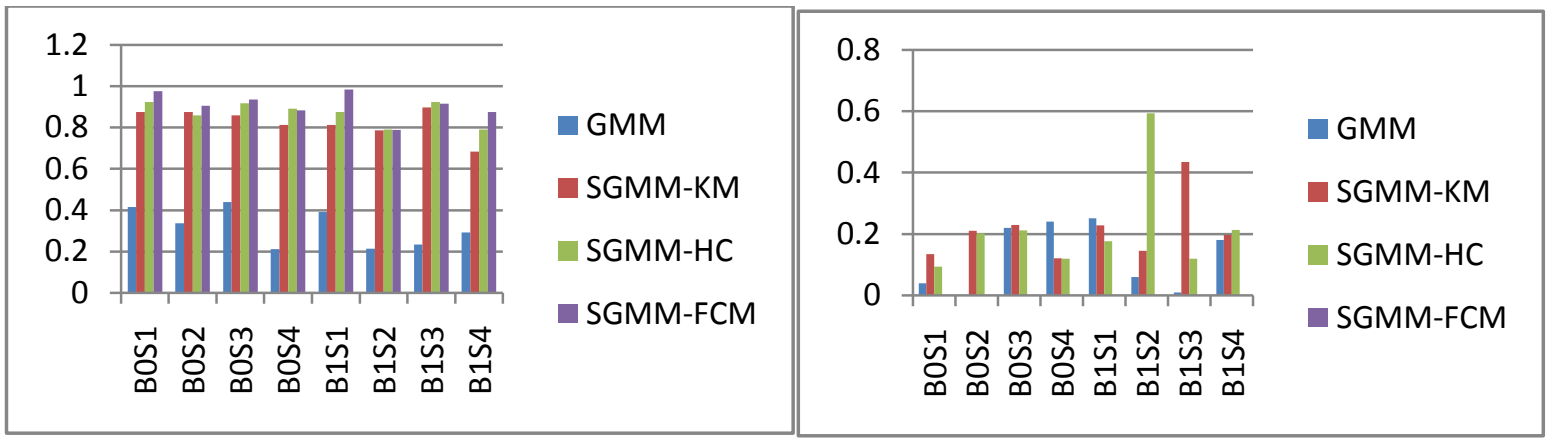

Figure C: Image Fidelity

Figure D: Mean Squared Error

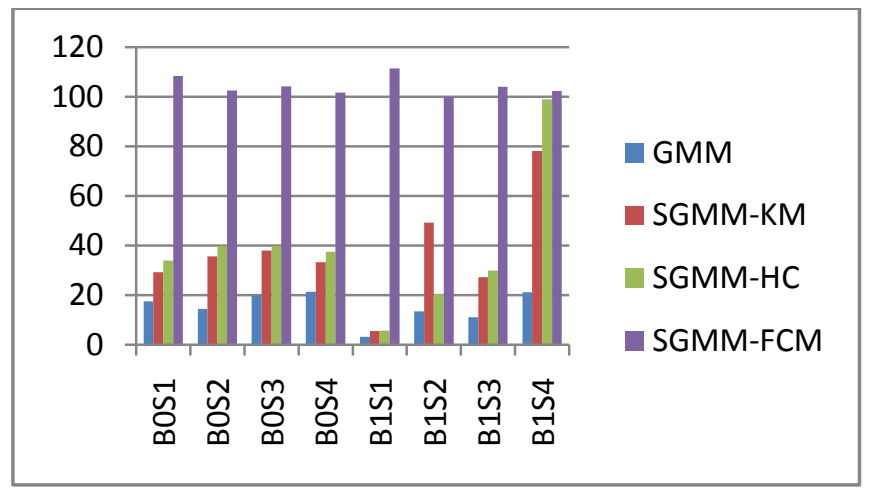

Figure E: Signal - to - Noise Ratio

Graphs - 2: Comparison of Techniques 
From the above Table -6 and bar graphs -2 , it can be clearly seen that the model developed by using hierarchical clustering shows better results with respect to the quality metrics. The model is compared the existing models based on Gaussian Mixture Model and Skew Gaussian Mixture Model with K - Means algorithm and the results are shown pictorially by the graphs -1 and graphs -2 .

From the above graphs, it can be clearly seen that the model developed by using hierarchical clustering performs better compared to the earlier models. This may be due to the fact of the asymmetric nature of the medical images.

\section{CONCLUSION}

In this paper, a medical image segmentation technique based on finite skew Gaussian mixture model with hierarchical clustering using EM algorithm is developed and evaluated. The results obtained by this algorithm outperform the existing methods. This method can be mainly suited in particular cases of medical pathology where diseases like acoustic neuroma and Parkinson's diseases can be identified accurately there by helping in proper diagnosis and preventing disabilities such as hearing loss and preventing disabilities such as hearing loss and dizziness.

\section{REFERENCES}

[1] Yamazaki T.: "Introduction of EM algorithm into color Image Segmentation"Proceedings of ICIRS'98, pp.368371. (1998)

[2] Srinivas Yarramalle, K.Srinivasa Rao: "Unsupervised image segmentation using finite doubly truncatedGaussianmixture model and hierarchical clustering” CURRENT SCIENCE, pp. 71 - 84. (2007).

[3]. Timor $\mathrm{K}$ and Michael Brady: "Unsupervised Nonparametric segmentation using level sets", Proceedings of $9^{\text {th }}$ IEEE International conference on Computer Vision (ICCV'03).

[4] Eskicioglu, A.M, et al "Image Quality measures and their performance",IEEE Transaction. Commum.1993,43.

[5] Nagesh Vadaparthi, Srinivas Yerramalle, and Suresh Varma.P: Unsupervised Medical Image Segmentation on Brain MRI images using Skew Gaussian Distribution, IEEE - ICRTIT 2011, pp.1293 - 1297.

[6]. D. L. Pham, C. Y. Xu, and J. L. Prince, "A survey of current methodsin medical image segmentation," Annu. Rev. Biomed.Eng., vol. 2, pp.315-337, 2000.

[7]. K. Van Leemput, F. Maes, D. Vandeurmeulen, and P. Suetens, "Automatedmodel-based tissue classification of MR images of the brain", IEEE Trans. Med. Imag., vol. 18 , no. 10, pp. 897-908, Oct. 1999.

[8]. G. Dugas-Phocion, M. Á. González Ballester, G. Malandain, C. Lebrunand N. Ayache, "Improved EM- based tissue segmentation andpartial volume effect quantification in multi-sequence brain MRI," inInt. Conf. Med. Image Comput.Comput. Assist. Int. (MICCAI), 2004,pp. 26-33

[9]. K. Van Leemput, F. Maes, D. Vandermeulen, and P. Suetens, "A unifyingframework for partial volume segmentation of brainMRimages", IEEE Trans. Med. Imag., vol. 22, no. 1, pp. 105-119, Jan. 2003.

[10]. M. Prastawa, E. Bullitt, S. Ho, and G. Gerig, "Robust estimation forbrain tumor segmentation," in Int. Conf. Med. Image Comput. Comput.Assist. Inter (MICCAI), 2003, pp. 530-537.

[11]. Sylvain Bouix et al. "Evaluating Brain Tissue Classifiers without a ground truth", Journal of NeuroImage (ELSEVIER) - 36, pp. 1207 - 1224, 2007.

[12] Ahmet M. Eskicioglu and Paul S. Fisher: "Image Quality Measures and Their Performance", IEEE Transactions on Communications, Vol. 43, No.12, Dec.'95, pp. $2959-2965$

\section{AUTHORS BIOGRAPHY}

Mr. V.Nagesh is an Associate Professor in the department of Information Technology, MVGR College of Engineering, Vizianagaram, Andhra Pradesh, India. He has 9 years of teaching experience and his areas of interests include Communication Networks \& Protocols, Grid Computing and Bioinformatics apart from Image processing. He has published several research papers in National and International level. He is a life member of CSI and ISTE.

Dr. Y.Srinivas is a Professor in the department of Information Technology, GITAM University, Visakhapatnam, India and has about 17 years of teaching experience. His areas of interests include Speech Processing, Data mining, and Software reusability apart from Image processing. He is having more than 60 publications at national and International level. He is the author for 4 books and a life member of ISTE, CSI, IE, ISTAM, IISA and ISPS.

Dr. P .Suresh Varma is a Professor in the department of Computer Science and Principal of Adikavi Nannaya University, Rajahmundry, India. He is having 17 years of teaching experience. His areas of interests include Communication Networks, Image Processing and Speech Processing. He has published several research papers. He is a life member of ISTE, SMORSI, ISCA and IISA.

Dr. P.S.R.Murthy is an Associate Professor in the department of Mathematics, GITAM University, Visakhapatnam, India. He has 17 years of teaching experience and published several papers. He is a life member of IACSIT and his research areas include Fluid Dynamics, Lattice Theory and Mathematical Modeling. 